\title{
Some Test Procedures and Their Performance under Umbrella Alternatives
}

\author{
Sanhita Sarmah ${ }^{1}$ and Bipin Gogoi ${ }^{2}$ \\ Research Scholar, Department of Statistics, Dibrugarh University ${ }^{1}$ \\ Professor, Dept. of Statistics, Dibrugarh University ${ }^{2}$
}

\begin{abstract}
Performance of Mack-Wolfe type test using different scores suitable for long tail, short tail, right skewed distributions under Umbrella alternatives are studied. We also compare the performance of this type test with the Jonckheere test of ordered alternative and general classical F- statistic considering various combinations of parameters. Results are obtained by simulation technique repeating 10,000 times. These results are presented in different tables. Results are also shown graphically for easy visual comparison.
\end{abstract}

Key Words: Umbrella Alternatives, F-test, Jonchkerre test, Mack-Wolfe type test, Simulation, Robustness, Power.

\section{INTRODUCTION}

Test procedures for k-sample problem, where the generalized their work to allow for arbitrary scoring alternatives of interest are of the form schemes but were still concerned primarily with ordered $F_{1}(x) \geq F_{2}(x) \geq \ldots \geq F_{l-1}(x) \geq F_{l}(x) \leq F_{l+1}(x) \leq \ldots \leq$ alfernatives. Mack and Wolfe(1981) proposed a for all $\mathrm{x}$, with at least one strict inequality are referred to as umbrella alternatives, and the point(peak) $l$ is known as the umbrella point.

Let $\mathrm{X}_{\mathrm{i} 1}, \ldots, \mathrm{X}_{\mathrm{in}}, \mathrm{i}=1, \ldots, \mathrm{k}$, be the $\mathrm{k}$ independent random samples, with $\mathrm{X}_{\mathrm{ij}}, \mathrm{j}=1, \ldots, \mathrm{n}_{\mathrm{i}}$, having absolutely continuous distribution function $\mathrm{F}_{\mathrm{i}}(\mathrm{x})$. For such $\mathrm{k}$-sample data settings we are often interested in testing the hypothesis that all $\mathrm{k}$ samples came from a single common distribution; that is , the appropriate null hypothesis is Ho : $\mathrm{F}_{1}(\mathrm{x})=\ldots=\mathrm{F}_{\mathrm{k}}(\mathrm{x})$. A variety of tests procedures are available for this problem. Although the decision of selecting one such test to use is not as clearly specified as under the usual classical (normal theory) assumptions, we can narrow the potential distribution-free candidate by using any a priori information we might have about the alternative(to $\mathrm{Ho}$ ) that is of primary importance. For example, when the alternative to Ho is simply the general case that at least two of the $\mathrm{k}$ underlying distributions have different medians, then the most commonly used distribution free test procedure is that of Kruskal Wallis(1952). The Kruskal-Wallis test cannot be use any a priori information about possible relationships between the $\mathrm{k}$ population medians.

On the otherhand, if an experimenter has some a priori information concerning the possible alternatives to the null hypothesis of $\mathrm{k}$ identical distributions, he or she would do better to choose a distribution-free test that is designed for detecting these alternatives. The initial procedures of this type were designed for monotonically ordered alternatives of the form $\mathrm{H}_{1}$ : $F_{1}(x) \geq F_{2}(x) \geq \ldots \geq F_{l}(x) \leq \ldots \leq F_{k}(x)$ for all $\mathrm{x}$, with at least one strict inequality for at least one $\mathrm{x}$ value. Jonckheere(1954) and Terpstra(1952) were the independently proposed equivalent procedures. Puri(1965) and Archambault, Mack and Wolfe(1978) distribution -free procedures designed for umbrella alternatives. Alternatives of this type are appropriate, for example, the Wechsler Adult Intelligence Scale(WAIS) scores for pictures completion can be assumed to have an up-than-down umbrella pattern with respect to increasing age [Wechsler(1958) and Pan(1996)].Some other example , in evaluating marginal gain in performance efficiency as a function of time, crop yield as function of quantity fertilizer applied, reaction to increasing drug dosage levels, and effect of age on responses to certain stimuli, to name but a few. In such examples downturn are possible after certain point of time, dosage etc.

\section{MODEL AND THE HYPOTHESES}

Let $\mathrm{X}_{\mathrm{i} 1}, \ldots, \mathrm{X}_{\mathrm{in}}{ }_{i}, \mathrm{i}=1, \ldots, \mathrm{k}$, be independent random samples from populations with absolutely continuous distribution functions $\mathrm{Fi}(\mathrm{x})=\mathrm{F}\left(\mathrm{x}-\theta_{i}\right), \mathrm{i}=1, \ldots, \mathrm{k}$. We wish to test:

Ho : $\theta_{1}=\ldots=\theta_{k}$

Against the umbrella alternative

$\mathrm{H}_{1}=\theta_{1} \leq \ldots \leq \theta_{l} \geq \ldots \geq \theta_{k}$

With at least strict inequality, where, at first, 1 is assumed to be known.

\section{THE TEST STATISTICS}

\subsection{F- Test}

The F statistic is defined by

$$
\mathrm{F}=\frac{\sum_{i=1}^{k} n_{i}\left(x_{i}-\bar{x}\right)^{2} /(k-1)}{\sum_{i=1}^{k}\left(n_{i}-1\right) s_{i}^{2} /(N-k)} \quad \ldots
$$




$$
\begin{aligned}
& \text { where } \mathrm{N}=\sum_{i} \quad \mathrm{n}_{\mathrm{i}}, \quad x_{i}=\sum_{j} \mathrm{x}_{\mathrm{ij}} / \mathrm{n}_{\mathrm{i}}, \\
& \bar{x}=\sum_{i} \sum_{j} \mathrm{x}_{\mathrm{ij}} / \mathrm{N}=\sum_{i} \mathrm{n}_{\mathrm{i}} \bar{x}_{i} / \mathrm{N}, \\
& \mathrm{s}_{\mathrm{i}}^{2}=\sum_{j} \quad\left(\mathrm{x}_{\mathrm{ij}}-\bar{x}_{i}\right)^{2} /\left(\mathrm{n}_{\mathrm{i}}-1\right) \\
& i=1,2, \ldots, k ; j=1,2, \ldots, n_{i}
\end{aligned}
$$

under $\mathrm{H}_{0}$, the statistic $\mathrm{F}$ has an F-distribution with $\mathrm{k}-1$ and $\mathrm{N}-\mathrm{k}$ degrees of freedom. If we assume nonnormal distribution with at least finite second moments it can be shown that under $\mathrm{H}_{0}, \mathrm{~F}$ has asymptotically a chi-square distribution with k-1 degrees of freedom as shown by Tiku(1986).

\subsection{Jonckheere Test:}

Let

$$
\mathrm{U}_{\mathrm{iljm}}=\left[\begin{array}{l}
1, \text { if } \mathrm{x}_{\mathrm{il}}<\mathrm{x}_{\mathrm{jm}} \\
0, \text { if } \mathrm{x}_{\mathrm{il}}>\mathrm{x}_{\mathrm{jm}}
\end{array}\right.
$$

where $\mathrm{i}=1,2, \ldots, \mathrm{k}-1 ; \mathrm{j}=\mathrm{i}+1, \ldots, \mathrm{k}$;

$$
1=1,2, \ldots, n_{i} ; j=1,2, \ldots, n_{j} \text {. }
$$

Let

$$
\mathrm{U}_{\mathrm{ij}}=\sum_{i l=1}^{n_{i}} \sum_{m=1}^{n_{j}} u_{i j j m}
$$

And finally, we have the Jonckheere statistic of the form

$$
\mathrm{S}=2 \sum_{i=1}^{k-1} \sum_{j=i+1}^{k} u_{i j j m}-\sum_{i=1}^{k-1} \sum_{j=i+1}^{k} n_{i} n_{j} \ldots
$$

It follows that for large samples we may in most cases assume

$$
\begin{aligned}
& \frac{S}{\left.\sqrt{\left[\frac{1}{18}\right.}\left\{N^{2}(2 N+3)-\sum n_{i}^{2}\left(2 n_{i}+3\right)\right\}\right]} \\
& \text { where } \mathrm{N}=\sum_{i=1}^{k} n_{i}
\end{aligned}
$$

to have a standard normal distribution. Since the interval between all possible adjascent values of $\mathrm{S}$ is always two, an improvement in the approximation to the true distribution will be obtained if unity is subtracted from an obtained value of $\mathrm{S}$, prior to its division by the standard deviation.

So under Ho test s reject if

$$
\left.\mathrm{S} \geq 1+Z_{\alpha} \sqrt{\left[\frac{1}{18}\right.}\left\{N^{2}(2 N+3)-\sum_{i=1}^{k} n_{i}^{2}\left(2 n_{i}+3\right)\right\}\right]
$$

When $n_{i}=n$,

Under Ho test $\mathrm{S}$ is reject if

$\mathrm{S} \geq 1+z_{\alpha}\left[\left(N^{2}(2 N+3)-k\left(\mathrm{n}^{2}\right)(2 \mathrm{n}+3)\right) / 18\right]^{1 / 2}$

provided $\mathrm{N} \geq 15$.

\subsection{Mack-Wolfe tests}

Now we consider the well-known test of Mack and Wolfe(1981), the test statistic of which is based on twosample statistics of Mann and Whitney(1947) given by

$$
\begin{gathered}
\mathrm{Urs}=\sum_{h=1}^{n_{r}} \sum_{j=1}^{n_{s}} W_{h j} \text { for the rth and sth sample, } 1 \\
\leq r<s \leq k \text {, with } \\
\qquad \mathrm{W}_{\mathrm{hj}}=\left[\begin{array}{l}
1, \text { if } \mathrm{x}_{\mathrm{rk}}<\mathrm{x}_{\mathrm{sj}} \\
0, \text { otherwise }
\end{array}\right.
\end{gathered}
$$

The Mack-Wolfe test statistic MW is then defined by

$$
\mathrm{MW}=\sum_{r=1}^{l-1} \sum_{s=r+1}^{l} U_{r s}+\sum_{r=l}^{k-1} \sum_{s=r+1}^{k} U_{s r}
$$

Under Ho, we have

$$
\begin{gathered}
\mathrm{E}(\mathrm{MW})=\frac{1}{4}\left(m_{1}^{2}+m_{2}^{2}-\sum_{i=1}^{k} n_{i}^{2}-n_{l}^{2}\right) \\
\text { And } \operatorname{Var}(\mathrm{MW})=
\end{gathered}
$$

$\frac{1}{72}\left[2\left(m_{1}^{3}+m_{2}^{3}\right)+3\left(m_{1}^{2}+m_{2}^{2}\right)\right.$

$\left.-\sum_{i=1}^{k} n_{1}^{2}\left(2 n_{i}+3\right)-n_{l}{ }^{2}\left(2 n_{l}+3\right)+12 n_{l} m_{1} m_{2}-12 n_{l}{ }^{2} N\right]$

With $\mathrm{N}=\sum_{i=1}^{k} n_{i}, \mathrm{~m}_{1}=\sum_{i=1}^{l} n_{i}$ and

$\mathrm{m}_{2}=\sum_{l i=l}^{k} n_{i}$

It is easy to see that ${ }^{k-1}$ MW $=$
$\sum_{i=1}^{l} U_{(1 \ldots i-1) i}+\sum_{i=l}^{k-1} U_{(k \ldots i+1) i}$, where $U_{(1 \ldots i-1) i}, 2 \leq i \leq l$

,is the two-sample Mann-Whitney statistic computed on the ith sample versus the combined data in the first $\mathrm{i}-1$ samples and analogously $U_{\left(c_{\ldots i+1) i}\right.}, l \leq i \leq k-1$, the two-sample Mann-Whitney statistic computed on the ith sample versus the combined data in the last $\mathrm{k}-1$ samples . Buning and Kossler(1997) generalized the MW-test by replacing the Mann-Whitney statistic in the formula for MW by other two-sample statistics, e.g. by those of Gastwirth(1965) and Hogg et. al.(1975).

The general form of the two-sample linear rank statistic is given by $\left(\mathrm{N}_{\mathrm{rs}}=\mathrm{n}_{\mathrm{r}}+\mathrm{n}_{\mathrm{s}}\right)$ :

$$
\mathrm{L}_{\mathrm{rs}}=\sum_{p=1}^{N_{r s}}\left[a\left(N_{r s}, p\right)-\bar{a}\left(N_{r s}\right)\right] V_{p}
$$

With $a\left(N_{r s}, p\right) \in \Re, \quad \bar{a}\left(N_{r s}\right)=\frac{1}{N_{r s}} \sum_{p=1}^{N_{r s}} a\left(N_{r s}, p\right)$

$$
V_{p}=\left[\begin{array}{l}
1, \text { if } X_{p} \text { belong to the sth sample } \\
0,
\end{array}\right.
$$


where $X(p)$ is the pth order statistic of combined two samples $\mathrm{X}_{\mathrm{r}_{1}}, \ldots, \mathrm{X}_{\mathrm{rn}}{ }_{r}$ and $\mathrm{X}_{\mathrm{s}_{1}}, \ldots, \mathrm{X}_{\mathrm{sn}_{s}}$. Then MackWolfe type statistics are defined by

$$
\mathbf{M W T}=\sum_{i=2}^{l} N_{i} L_{(1 \ldots i-1) i}+\sum_{i=l}^{k-1}\left(N-N_{i-1}\right) L_{(k \ldots i+1) i}
$$

Where $\quad N_{i}=\sum_{j=1} n_{j}, \mathrm{~N}=\mathrm{N}_{\mathrm{c}}$, and $L_{(1 \ldots i-1) i}$ is an given by

arbitrary two-sample linear rank statistic computed on the ith sample versus the combined data in the first i-1 samples and analogously $L_{(c \ldots i+1) i}$ is computed on the ith sample versus the combined data in the last k-i samples.

Let us now consider some examples of Mack-Wolfe type tests with specially chosen two-samples scores. In the parenthesis that type of distribution is indicated for which the test has high power.

\subsection{Gastwirth test $\mathbf{G}$ (short tails)}

$$
\mathrm{a}_{\mathrm{G}}\left(\mathrm{N}_{\mathrm{rs}}, \mathrm{p}\right)=\frac{4}{\mathrm{~N}_{\mathrm{rs}}+1}\left[\begin{array}{c}
\mathrm{p}-\frac{\mathrm{N}_{\mathrm{rs}}+1}{4}, \text { if } \mathrm{p} \leq \frac{\mathrm{N}_{\mathrm{rs}}+1}{4} \\
0, \text { if } \frac{\mathrm{N}_{\mathrm{rs}}+1}{4}<p<\frac{3\left(\mathrm{~N}_{\mathrm{rs}}+1\right)}{4} \\
\mathrm{p}-\frac{3\left(\mathrm{~N}_{\mathrm{rs}}+1\right)}{4}, \text { if } \mathrm{p} \geq \frac{3\left(\mathrm{~N}_{\mathrm{rs}}+1\right)}{4}
\end{array}\right.
$$

\subsection{The test LT (Long Tails)}

$\mathrm{a}_{\mathrm{LT}}\left(\mathrm{N}_{\mathrm{rs}}, \mathrm{p}\right)=\left[\begin{array}{c}-1, \quad \text { if } \mathrm{p}<\frac{\mathrm{N}_{\mathrm{rs}}}{4}+1 \\ \frac{4 \mathrm{p}}{\mathrm{N}_{\mathrm{rs}}+1}-2, \text { if } \frac{\mathrm{N}_{\mathrm{rs}}}{4}+1 \leq \mathrm{p} \leq \frac{3\left(\mathrm{~N}_{\mathrm{rs}}+1\right)}{4} \\ 1, \quad \text { if } \mathrm{p} \geq \frac{3\left(\mathrm{~N}_{\mathrm{rs}}+1\right)}{4}\end{array}\right.$

3.6 The - Hogg-Fisher Randles test HFR (right skewed)

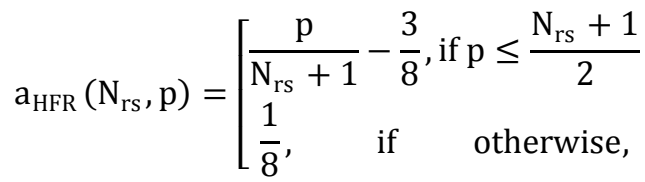

The Mack-Wolfe type tests based on Gastwirth scores, LT- scores and Hogg-Fisher-Randles scores are abbreviated by MWG, MWLT, and MWHFR, respectively. Under Ho, we have $\mathrm{E}(\mathrm{MWT})=0$ and the limiting distribution of MWT / $\sigma_{M W T}$ is, under some regularity conditions, the standard normal where the asymptotic variance of MWT, Var (MWT) $=\sigma_{M W T}^{2}$ is

$$
\begin{aligned}
\sigma_{M W T}^{2} & =\mathrm{I}(\mathrm{g})\left[\left(\mathrm{N}_{l}^{3}-\sum_{i=1}^{l} n_{i}^{3}+\left(N-N_{l-1}\right)^{3}-\sum_{i=l}^{k} n_{i}^{3}\right) / 3\right. \\
& \left.+2 \mathrm{~N}_{\mathrm{l}-1} \mathrm{n}_{\mathrm{i}}\left(\mathrm{N}-\mathrm{N}_{\mathrm{l}}\right)\right]
\end{aligned}
$$

$\mathrm{I}(\mathrm{g})$ is the Fisher Information and $\mathrm{g}$ is that density function for which the two-sample test is the definition of MWT is asymptotically most powerful(from Puri 1965). Specially for the MWG,MWLT and MWHFR statistic I $(\mathrm{g})$ takes the values $1 / 6,2 / 3$ and 5/192, respectively Buning and Kossler(1997).

\section{MONTE CARLO STUDY}

In this section we present the results of Monte Carlo power study comparing six tests for the c-sample location problem, namely, F test, Jonckheere(1964) J test, MackWolfe(1981) W test, and three Mack-Wolfe type tests short-tailed MWS, long -tailed MWL and right skewed MWR with umbrella point $\mathrm{p}$ known. The relative powers were studied for location shift alternatives and 5,10,20 observations for each $\mathrm{k}=3$ or 4 or 5 treatments, at the nominal significance levels of .01, .05 and .10. We considered seven different sampling distributions, namely, normal, uniform, lognormal, Logistic, exponential, double exponential and Cauchy. The observations are drawn from the distributions using integral transform method. We have use uniform number generators RND (uniform $(0,1)$ ) and Box-Muller formula(1958) for standard normal deviate. In table 3.1, 3.2, 3.3, 3.4, 3.5, 3.6, 3.7 we present the estimated power of the tests under different distributions considering the different sets of location parameters Note

\begin{tabular}{|c|c|c|c|c|c|c|c|c|c|}
\hline \multirow{2}{*}{$\begin{array}{l}\text { Sample } \\
\text { Number k }\end{array}$} & \multirow{2}{*}{$\begin{array}{l}\text { Pick } \\
1\end{array}$} & \multirow{2}{*}{$\begin{array}{l}\text { Number of } \\
\text { Observation } n_{i}\end{array}$} & \multirow{2}{*}{$\begin{array}{l}\text { Effects } \\
\mu_{i}\end{array}$} & \multicolumn{5}{|c|}{ Test Statistics } & \multirow[b]{2}{*}{ MWR } \\
\hline & & & & $\mathrm{F}$ & $\mathrm{J}$ & MW & MWS & MWL & \\
\hline \multirow[t]{11}{*}{3} & 2 & $5,5,5$ & $\begin{array}{lll}0 & 0 & 0\end{array}$ & .0485 & .0443 & .0398 & .0000 & .0487 & .0269 \\
\hline & 2 & & $\begin{array}{lll}0 & .5 & 0\end{array}$ & 1004 & .0390 & .1104 & . 0000 & .1205 & .0483 \\
\hline & 2 & & $\begin{array}{lll}0 & .8 & 0\end{array}$ & 1934 & .0329 & .2242 & .0000 & .2358 & .0812 \\
\hline & 2 & & $\begin{array}{lll}0 & 1 & 0\end{array}$ & 2769 & .0289 & .3308 & .0000 & .3458 & .1583 \\
\hline & 2 & & $\begin{array}{lll}.1 & .5 & .1\end{array}$ & .0832 & .0420 & .0847 & .0000 & .0933 & .2313 \\
\hline & 2 & $10,10,10$ & $\begin{array}{lll}0 & 0 & 0\end{array}$ & .0506 & 0539 & .0474 & .0230 & .0506 & .0387 \\
\hline & 2 & & $\begin{array}{lll}0 & .5 & 0\end{array}$ & .1710 & .0479 & .2168 & .1146 & .2022 & .1616 \\
\hline & 2 & & $\begin{array}{lll}0 & .8 & 0\end{array}$ & .3882 & .0411 & .4745 & .2857 & .4448 & .3605 \\
\hline & 2 & & $\begin{array}{lll}0 & 1 & 0\end{array}$ & .5681 & .0355 & .6597 & .4446 & .6226 & .5224 \\
\hline & 2 & & .1 .5 .1 & .1250 & .0500 & .1545 & .0801 & .1434 & .1176 \\
\hline & 2 & $20,20,20$ & $\begin{array}{lll}0 & 0 & 0\end{array}$ & .0465 & .0528 & .0484 & .0358 & .0483 & .0426 \\
\hline
\end{tabular}
that the " 0 " shift corresponds to the standard forms for the all distributions considered. To estimate the each result we have repeated 10,000 times.

Table1 Empirical level and power of six tests under Normal Distribution 
IARJSET

International Advanced Research Journal in Science, Engineering and Technology

Vol. 3, Issue 1, January 2016

\begin{tabular}{|c|c|c|c|c|c|c|c|c|c|}
\hline \multirow[b]{2}{*}{4} & $\begin{array}{l}2 \\
2 \\
2 \\
2\end{array}$ & & $\begin{array}{lrl}0 & .5 & 0 \\
0 & .8 & 0 \\
0 & 1 & 0 \\
.1 & .5 & .1\end{array}$ & $\begin{array}{l}.3256 \\
.7160 \\
.9004 \\
.2159\end{array}$ & $\begin{array}{l}.0493 \\
.0427 \\
.0372 \\
.0500\end{array}$ & $\begin{array}{l}.4163 \\
.7992 \\
.9371 \\
.2861\end{array}$ & $\begin{array}{l}.3268 \\
.7151 \\
.8882 \\
.2176\end{array}$ & $\begin{array}{l}.3747 \\
.7405 \\
.9052 \\
.2593\end{array}$ & $\begin{array}{l}.3200 \\
.6804 \\
.8560 \\
.2161\end{array}$ \\
\hline & $\begin{array}{l}2 \\
3 \\
3 \\
3 \\
3\end{array}$ & $5,5,5,5$ & $\begin{array}{l}0,0,0,0 \\
0, .5,1, .5 \\
0, .3,1, .8 \\
0, .5,1,0 \\
0,0, .5,0\end{array}$ & $\begin{array}{l}.0495 \\
.1892 \\
.2266 \\
.2476 \\
.0960\end{array}$ & $\begin{array}{l}.0488 \\
.2155 \\
.3988 \\
.0610 \\
.0711\end{array}$ & $\begin{array}{l}.0482 \\
.2560 \\
.2019 \\
.3534 \\
.1194\end{array}$ & $\begin{array}{l}.0100 \\
.0815 \\
.0572 \\
.1123 \\
.0270\end{array}$ & $\begin{array}{l}.0542 \\
.2551 \\
.2021 \\
.3454 \\
.1264\end{array}$ & $\begin{array}{l}.0329 \\
.1634 \\
.1110 \\
.2336 \\
.0726\end{array}$ \\
\hline \multirow{6}{*}{5} & $\begin{array}{l}2 \\
2 \\
3 \\
2 \\
3 \\
2\end{array}$ & $10,10,10,10$ & $\begin{array}{l}0,0,0,0 \\
0, .8, .5,0 \\
0, .5, .8,0 \\
0, .5, .3,0 \\
0, .3, .5,0 \\
.1, .3, .2, .1\end{array}$ & $\begin{array}{l}.0451 \\
.3676 \\
.3661 \\
.1563 \\
.1583 \\
.0600\end{array}$ & $\begin{array}{l}.0465 \\
.0239 \\
.0595 \\
.0320 \\
.0565 \\
.0408\end{array}$ & $\begin{array}{l}.0505 \\
.4803 \\
.4865 \\
.2269 \\
.2296 \\
.0790\end{array}$ & $\begin{array}{l}.0257 \\
.3387 \\
.3397 \\
.1392 \\
.1477 \\
.0428\end{array}$ & $\begin{array}{l}.0499 \\
.4430 \\
.4471 \\
.2093 \\
.2094 \\
.0759\end{array}$ & $\begin{array}{l}.0394 \\
.3679 \\
.3722 \\
.1641 \\
.1661 \\
.0598\end{array}$ \\
\hline & $\begin{array}{l}2 \\
3 \\
2\end{array}$ & $15,15,15,15$ & $\begin{array}{l}0,0,0,0 \\
0,1, .5, .0 \\
0, .5,1, .5\end{array}$ & $\begin{array}{l}.0436 \\
.5647 \\
.7219\end{array}$ & $\begin{array}{l}.0544 \\
.4829 \\
.0137\end{array}$ & $\begin{array}{l}.0481 \\
.6815 \\
.8348\end{array}$ & $\begin{array}{l}.0352 \\
.5813 \\
.7484\end{array}$ & $\begin{array}{l}.0500 \\
.6325 \\
.7892\end{array}$ & $\begin{array}{l}.0419 \\
.5460 \\
.7161\end{array}$ \\
\hline & $\begin{array}{l}3 \\
3 \\
3 \\
3 \\
2\end{array}$ & $20,20,20,20$ & $\begin{array}{l}0,0,0,0 \\
0, .5,1, .5 \\
0, .3,1, .8 \\
0, .5,1,0 \\
.1,1, .5, .1\end{array}$ & $\begin{array}{l}.0491 \\
.7286 \\
.8345 \\
.8717 \\
.7692\end{array}$ & $\begin{array}{l}.0513 \\
.5791 \\
.9044 \\
.0989 \\
.0141\end{array}$ & $\begin{array}{l}.0503 \\
.8119 \\
.6978 \\
.9313 \\
.8660\end{array}$ & $\begin{array}{l}.0396 \\
.7422 \\
.6131 \\
.8845 \\
.8055\end{array}$ & $\begin{array}{l}.0496 \\
.7624 \\
.6440 \\
.8951 \\
.8245\end{array}$ & $\begin{array}{l}.0444 \\
.6884 \\
.5336 \\
.8442 \\
.7591\end{array}$ \\
\hline & $\begin{array}{l}2 \\
2 \\
3 \\
4\end{array}$ & $5,5,5,5,5$ & $\begin{array}{c}0,0,0,0,0,0 \\
.3,1, .8, .5,0 \\
0, .3, .5, .3,0 \\
0, .3, .5,1,0\end{array}$ & $\begin{array}{l}.0527 \\
.1350 \\
.0496 \\
.1493\end{array}$ & $\begin{array}{l}.0469 \\
.0079 \\
.0447 \\
.0930\end{array}$ & $\begin{array}{l}.0479 \\
.3455 \\
.1348 \\
.3757\end{array}$ & $\begin{array}{l}.0183 \\
.1631 \\
.0421 \\
.1758\end{array}$ & $\begin{array}{l}.0494 \\
.3242 \\
.1274 \\
.3501\end{array}$ & $\begin{array}{l}.0360 \\
.2174 \\
.0847 \\
.2159\end{array}$ \\
\hline & $\begin{array}{l}2 \\
2 \\
3 \\
4\end{array}$ & $10,10,10,10,10$ & $\begin{array}{c}0,0,0,0,0,0 \\
.3,1, .8, .5,0 \\
0, .3, .5, .3,0 \\
0, .3, .5,1,0\end{array}$ & $\begin{array}{l}.0509 \\
.3006 \\
.0792 \\
.3379\end{array}$ & $\begin{array}{l}.0495 \\
.0028 \\
.0464 \\
.1401\end{array}$ & $\begin{array}{l}.0491 \\
.6264 \\
.2301 \\
.6516\end{array}$ & $\begin{array}{l}.0318 \\
.4941 \\
.1481 \\
.5240\end{array}$ & $\begin{array}{l}.0495 \\
.5699 \\
.2095 \\
.5988\end{array}$ & $\begin{array}{l}.0430 \\
.4932 \\
.1689 \\
.4953\end{array}$ \\
\hline & $\begin{array}{l}2 \\
2 \\
3 \\
4\end{array}$ & $20,20,20,20,20$ & $\begin{array}{l}0,0,0,0,0 \\
.3,1, .8, .5,0 \\
0, .3, .5, .3,0 \\
0, .3, .5,1,0\end{array}$ & $\begin{array}{l}.0455 \\
.6660 \\
.1627 \\
.7197\end{array}$ & $\begin{array}{l}.0484 \\
.0010 \\
.0446 \\
.2211\end{array}$ & $\begin{array}{l}.0457 \\
.9015 \\
.4188 \\
.9234\end{array}$ & $\begin{array}{l}.0405 \\
.8515 \\
.3564 \\
.8824\end{array}$ & $\begin{array}{l}.0470 \\
.8578 \\
.3774 \\
.8868\end{array}$ & $\begin{array}{l}.0448 \\
.8182 \\
.3313 \\
.8280\end{array}$ \\
\hline
\end{tabular}

Table 2 Empirical level and power of six tests under Uniform Distribution

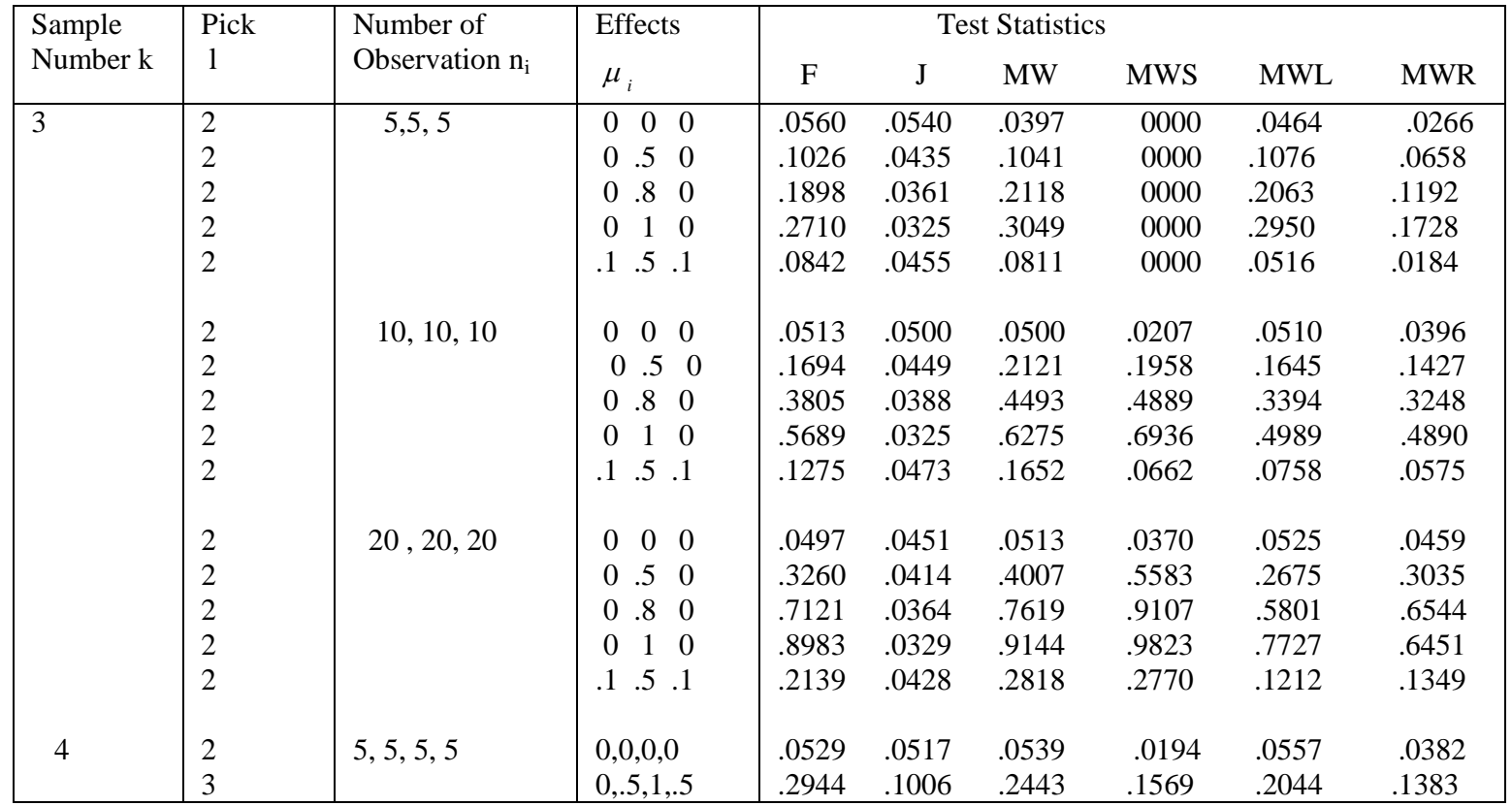


IARJSET

International Advanced Research Journal in Science, Engineering and Technology

Vol. 3, Issue 1, January 2016

\begin{tabular}{|c|c|c|c|c|c|c|c|c|c|}
\hline & $\begin{array}{l}3 \\
3 \\
3\end{array}$ & & $\begin{array}{l}0, .3,1, .8 \\
0, .5,1,0 \\
0,0, .5,0\end{array}$ & $\begin{array}{l}.5655 \\
.2414 \\
.1012\end{array}$ & $\begin{array}{l}.3603 \\
.0068 \\
.0316\end{array}$ & $\begin{array}{l}.1860 \\
.3224 \\
.1335\end{array}$ & $\begin{array}{l}.0730 \\
.2743 \\
.0648\end{array}$ & $\begin{array}{l}.1651 \\
.2734 \\
.1076\end{array}$ & $\begin{array}{l}.0947 \\
.1940 \\
.0568\end{array}$ \\
\hline & $\begin{array}{l}2 \\
2 \\
3 \\
2 \\
3 \\
2\end{array}$ & $10,10,10,10$ & $\begin{array}{l}0,0,0,0 \\
0, .8, .5,0 \\
0, .5, .8,0 \\
0, .5, .3,0 \\
0, .3, .5,0 \\
.1, .3, .2, .1\end{array}$ & $\begin{array}{l}.0488 \\
.3584 \\
.3565 \\
.1520 \\
.1523 \\
.0606\end{array}$ & $\begin{array}{l}.0501 \\
.0285 \\
.1260 \\
.0365 \\
.0636 \\
.0890\end{array}$ & $\begin{array}{l}.0518 \\
.4494 \\
.4567 \\
.2188 \\
.2148 \\
.0781\end{array}$ & $\begin{array}{l}.0294 \\
.3428 \\
.3452 \\
.1151 \\
.1166 \\
.0603\end{array}$ & $\begin{array}{l}.0516 \\
.2019 \\
.2003 \\
.0829 \\
.0799 \\
.0302\end{array}$ & $\begin{array}{l}.0453 \\
.1809 \\
.1780 \\
.0671 \\
.0634 \\
.0205\end{array}$ \\
\hline & $\begin{array}{l}2 \\
3 \\
2\end{array}$ & $15,15,15,15$ & $\begin{array}{l}0,0,0,0 \\
0,1, .5, .0 \\
0, .5,1, .5\end{array}$ & $\begin{array}{l}.0469 \\
.6921 \\
.6901\end{array}$ & $\begin{array}{l}.0514 \\
.3809 \\
.0335\end{array}$ & $\begin{array}{l}.0487 \\
.6702 \\
.8122\end{array}$ & $\begin{array}{l}.0328 \\
.8425 \\
.8500\end{array}$ & $\begin{array}{l}.0521 \\
.4729 \\
.4977\end{array}$ & $\begin{array}{l}.0493 \\
.5376 \\
.5312\end{array}$ \\
\hline & $\begin{array}{l}3 \\
3 \\
3 \\
2\end{array}$ & $20,20,20,20$ & $\begin{array}{l}0,0,0,0 \\
0, .5,1, .5 \\
0, .3,1, .8 \\
0, .5,1,0 \\
.1,1, .5, .1\end{array}$ & $\begin{array}{l}.0503 \\
.8627 \\
.4523 \\
.7822 \\
.7149\end{array}$ & $\begin{array}{l}.0487 \\
.7266 \\
.8958 \\
.3936 \\
.0568\end{array}$ & $\begin{array}{l}.0519 \\
.8068 \\
.6544 \\
.8785 \\
.8379\end{array}$ & $\begin{array}{l}.0418 \\
.9476 \\
.9060 \\
.9756 \\
.9104\end{array}$ & $\begin{array}{l}.0520 \\
.5752 \\
.4692 \\
.7362 \\
.5082\end{array}$ & $\begin{array}{l}.0479 \\
.6675 \\
.5226 \\
.8312 \\
.5901\end{array}$ \\
\hline 5 & $\begin{array}{l}3 \\
2 \\
3 \\
4\end{array}$ & $5,5,5,5,5$ & $\begin{array}{c}0,0,0,0,0,0 \\
.3,1, .8, .5,0 \\
0, .3, .5, .3,0 \\
0, .3, .5,1,0\end{array}$ & $\begin{array}{l}.0167 \\
.3241 \\
.1059 \\
.1961\end{array}$ & $\begin{array}{l}.0487 \\
.0019 \\
.0248 \\
.0301\end{array}$ & $\begin{array}{l}.0513 \\
.4496 \\
.1564 \\
.3856\end{array}$ & $\begin{array}{l}.0184 \\
.2590 \\
.0683 \\
.2825\end{array}$ & $\begin{array}{l}.0547 \\
.2603 \\
.1008 \\
.2658\end{array}$ & $\begin{array}{l}.0399 \\
.2119 \\
.0647 \\
.0522\end{array}$ \\
\hline & $\begin{array}{l}2 \\
2 \\
3 \\
4\end{array}$ & $10,10,10,10,10$ & $\begin{array}{c}0,0,0,0,0,0 \\
.3,1, .8, .5,0 \\
0, .3, .5, .3,0 \\
0, .3, .5,1,0\end{array}$ & $\begin{array}{l}.0489 \\
.5679 \\
.1530 \\
.3323\end{array}$ & $\begin{array}{l}.0506 \\
.0036 \\
.0487 \\
.1380\end{array}$ & $\begin{array}{l}.0505 \\
.5814 \\
.2215 \\
.6286\end{array}$ & $\begin{array}{l}.0289 \\
.5486 \\
.2296 \\
.7201\end{array}$ & $\begin{array}{l}.0493 \\
.4161 \\
.1519 \\
.4610\end{array}$ & $\begin{array}{l}.0466 \\
.4547 \\
.1496 \\
.4895\end{array}$ \\
\hline & $\begin{array}{l}2 \\
2 \\
3 \\
4\end{array}$ & $20,20,20,20,20$ & $\begin{array}{l}0,0,0,0,0 \\
.3,1, .8, .5,0 \\
0, .3, .5, .3,0 \\
0, .3, .5,1,0\end{array}$ & $\begin{array}{l}.0473 \\
.8605 \\
.2818 \\
.7152\end{array}$ & $\begin{array}{l}.0478 \\
.0076 \\
.1057 \\
.5382\end{array}$ & $\begin{array}{l}.0518 \\
.7355 \\
.3395 \\
.8861\end{array}$ & $\begin{array}{l}.0382 \\
.8214 \\
.4664 \\
.9721\end{array}$ & $\begin{array}{l}.0481 \\
.6961 \\
.2591 \\
.7342\end{array}$ & $\begin{array}{l}.0455 \\
.7989 \\
.3184 \\
.8319\end{array}$ \\
\hline
\end{tabular}

Table 3 Empirical level and power of six tests under Lognormal Distribution

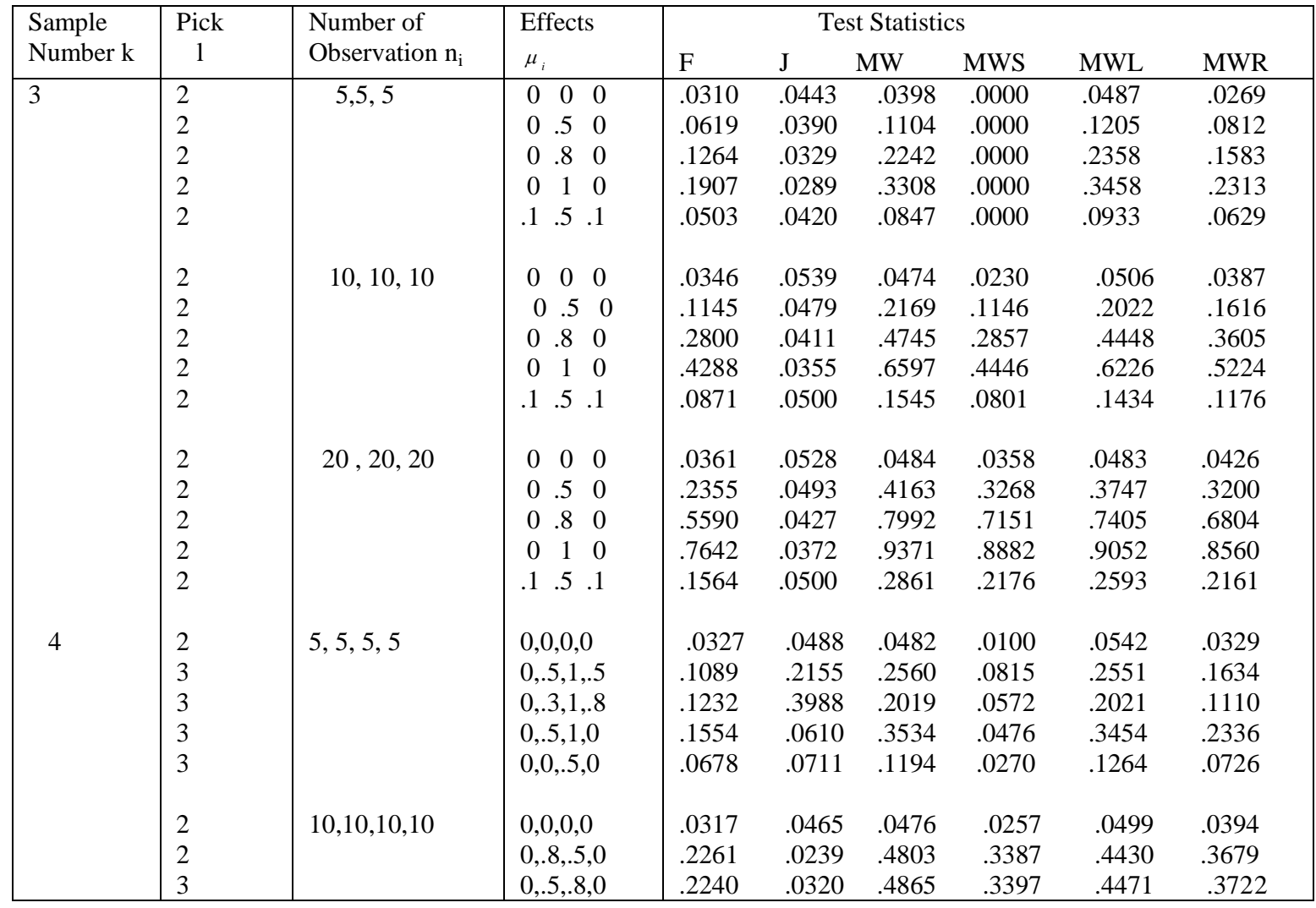


IARJSET

International Advanced Research Journal in Science, Engineering and Technology

Vol. 3, Issue 1, January 2016

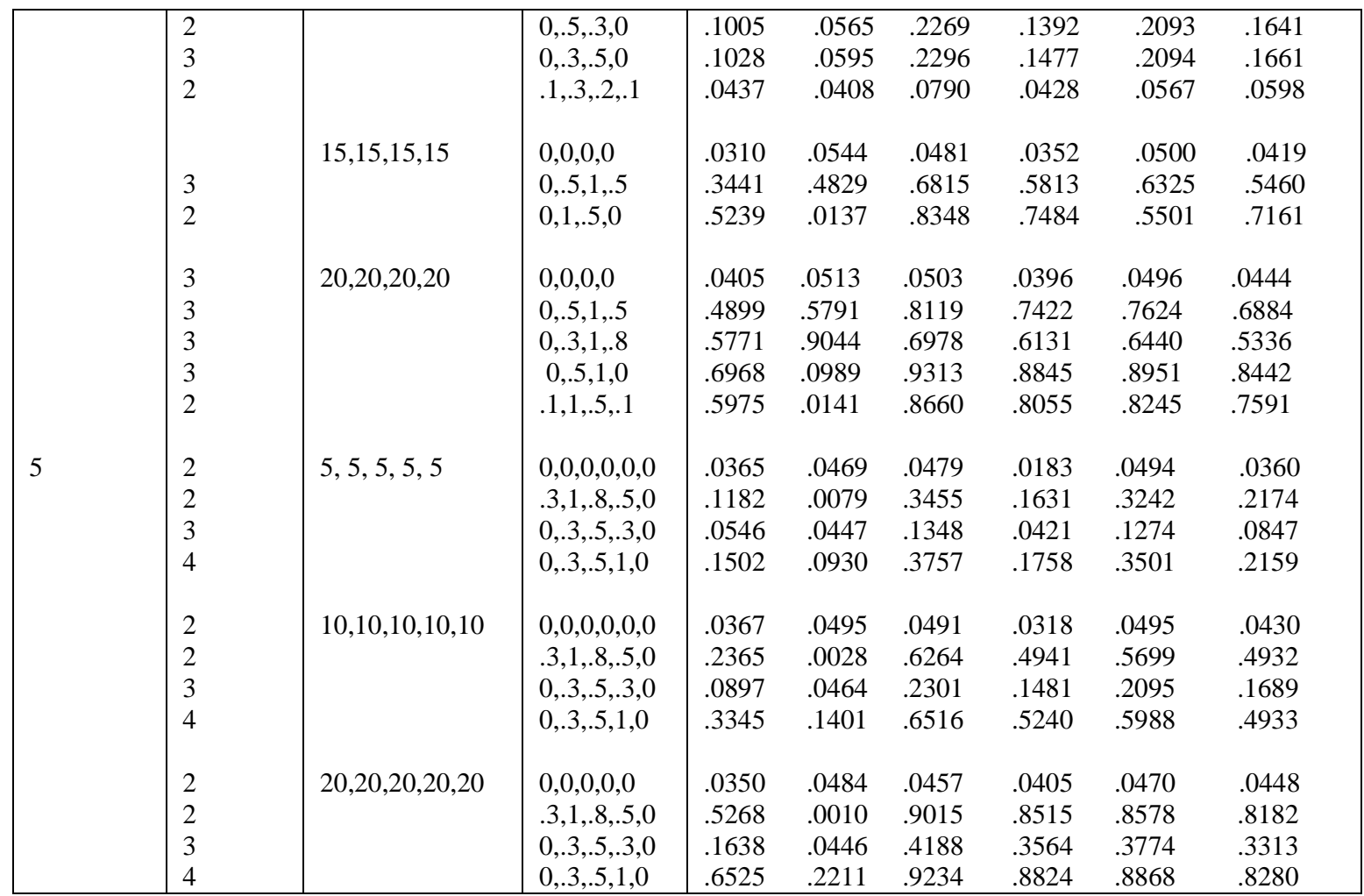

Table 4 Empirical level and power of six tests under Logistic Distribution

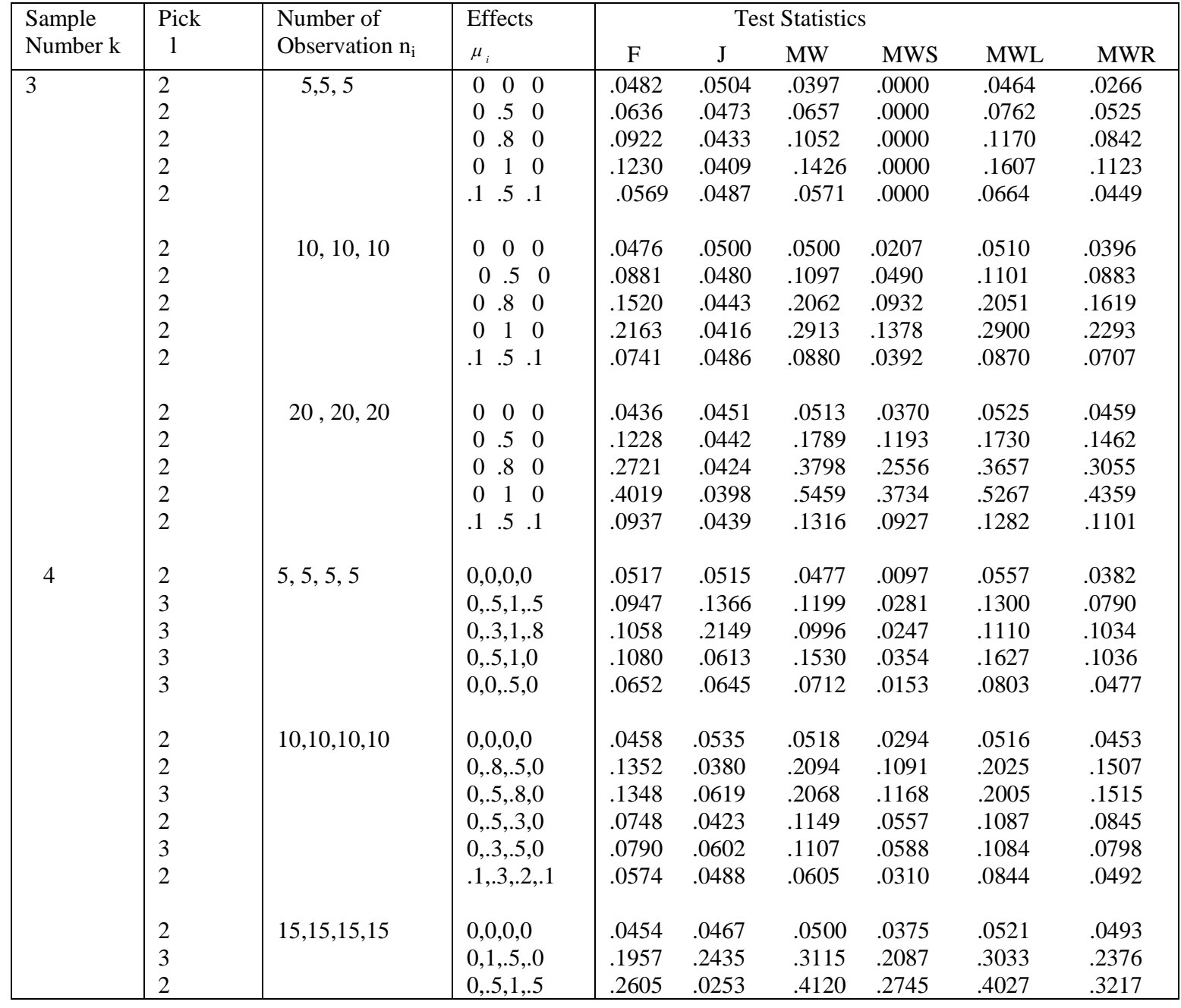


International Advanced Research Journal in Science, Engineering and Technology

Vol. 3, Issue 1, January 2016

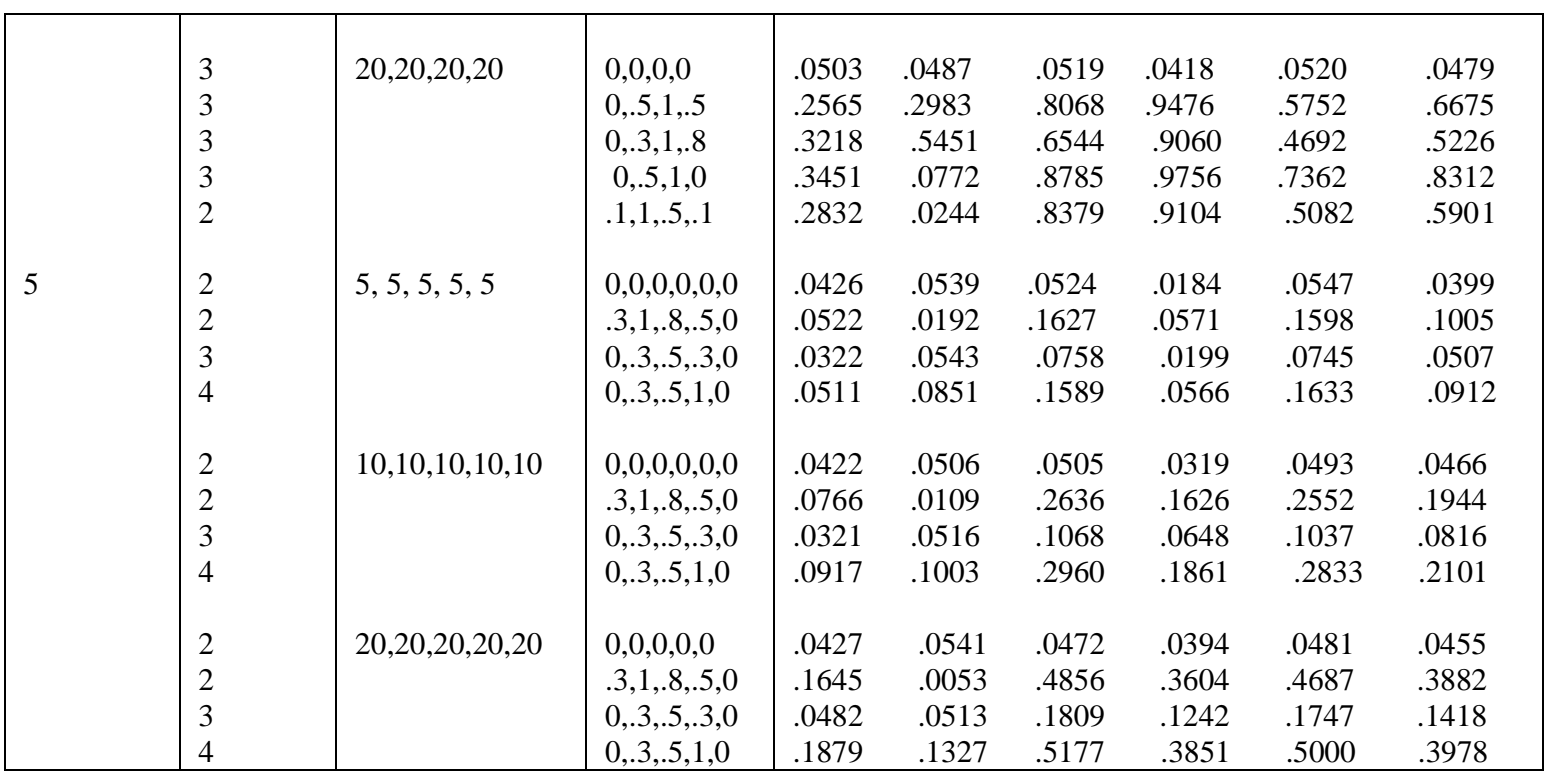

Table 5 Empirical level and power of six tests under Exponential Distribution

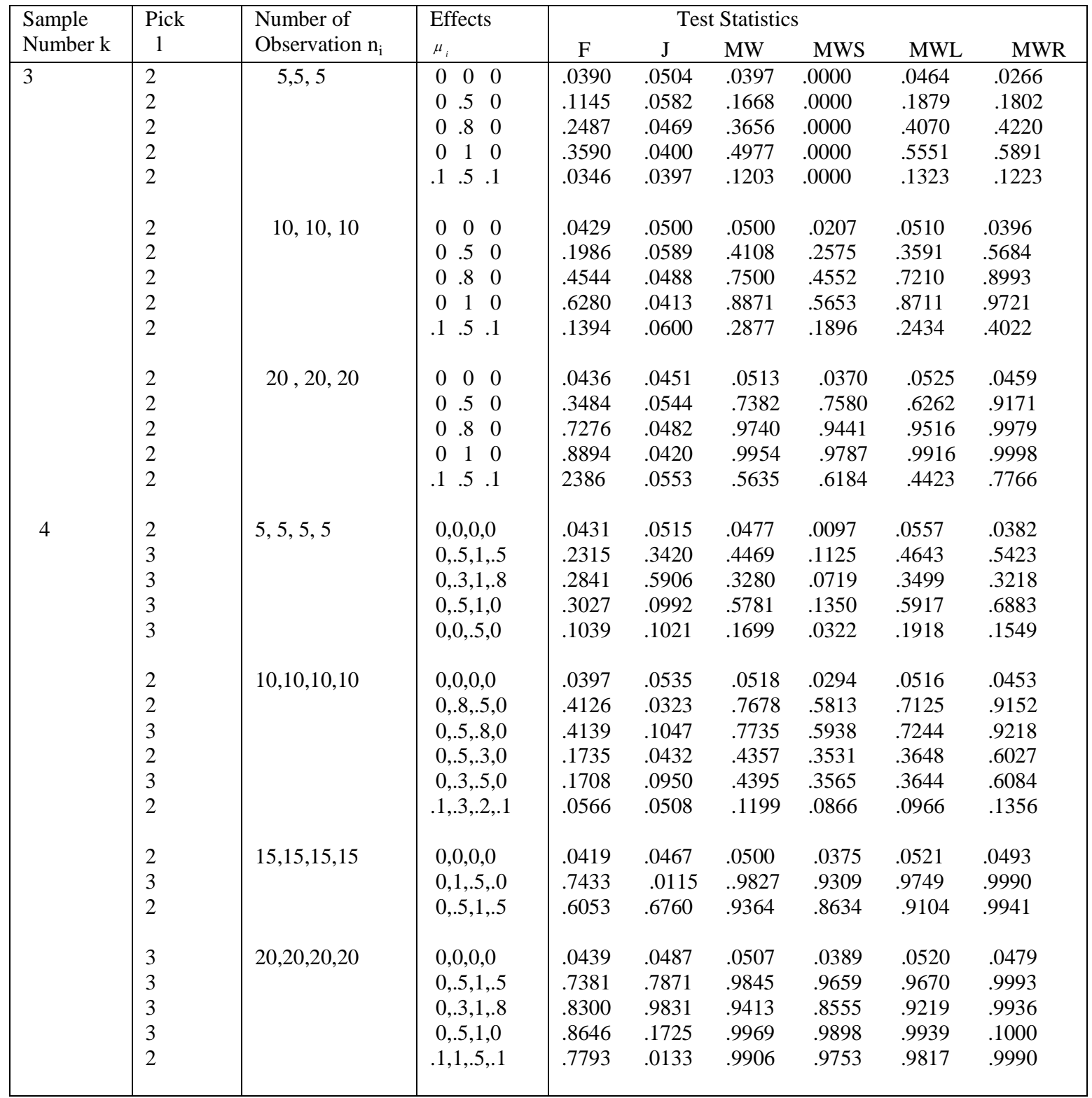


IARJSET

International Advanced Research Journal in Science, Engineering and Technology

Vol. 3, Issue 1, January 2016

\begin{tabular}{|c|c|c|c|c|c|c|c|c|c|}
\hline 5 & $\begin{array}{l}2 \\
2 \\
3 \\
4\end{array}$ & $20,20,20,20,20$ & $\begin{array}{l}0,0,0,0,0 \\
3,1, .8, .5,0 \\
0, .3, .5, .3,0 \\
0, .3, .5,1,0 \\
0,0,0,0,0,0 \\
.3,1, .8, .5,0 \\
0, .3, .5, .3,0 \\
0, .3, .5,1,0 \\
0,0,0,0,0 \\
.3,1, .8, .5,0 \\
0, .3, .5, .3,0 \\
0, .3, .5,1,0\end{array}$ & $\begin{array}{l}.0426 \\
.2465 \\
.0961 \\
.2616 \\
\\
.0422 \\
.4792 \\
.1536 \\
.5188 \\
\\
.0427 \\
.7925 \\
.2902 \\
.8385\end{array}$ & $\begin{array}{l}.0539 \\
.0075 \\
.0708 \\
.1717 \\
\\
.0506 \\
.0024 \\
.0689 \\
.2391 \\
\\
.0541 \\
.0002 \\
.0696 \\
.3674\end{array}$ & $\begin{array}{l}.0524 \\
.5829 \\
.2416 \\
.6122 \\
\\
.0505 \\
.8823 \\
.4557 \\
.9113 \\
\\
.0472 \\
.9949 \\
.7772 \\
.9975\end{array}$ & $\begin{array}{l}.0184 \\
.2450 \\
.0830 \\
.2451 \\
\\
.0319 \\
.7604 \\
.4081 \\
.7431 \\
\\
.0394 \\
.9941 \\
.8543 \\
.9919\end{array}$ & $\begin{array}{l}.0547 \\
.5716 \\
.2191 \\
.6169 \\
\\
.0493 \\
.8399 \\
.3750 \\
.8939 \\
\\
.0481 \\
.9849 \\
.6454 \\
.9953\end{array}$ & $\begin{array}{l}.0399 \\
.7252 \\
.2755 \\
.7190 \\
\\
.0466 \\
.9743 \\
.6386 \\
.9814 \\
\\
.0455 \\
.9998 \\
.9399 \\
1.000\end{array}$ \\
\hline
\end{tabular}

Table 6 Empirical level and power of six tests under Double Exponential Distribution

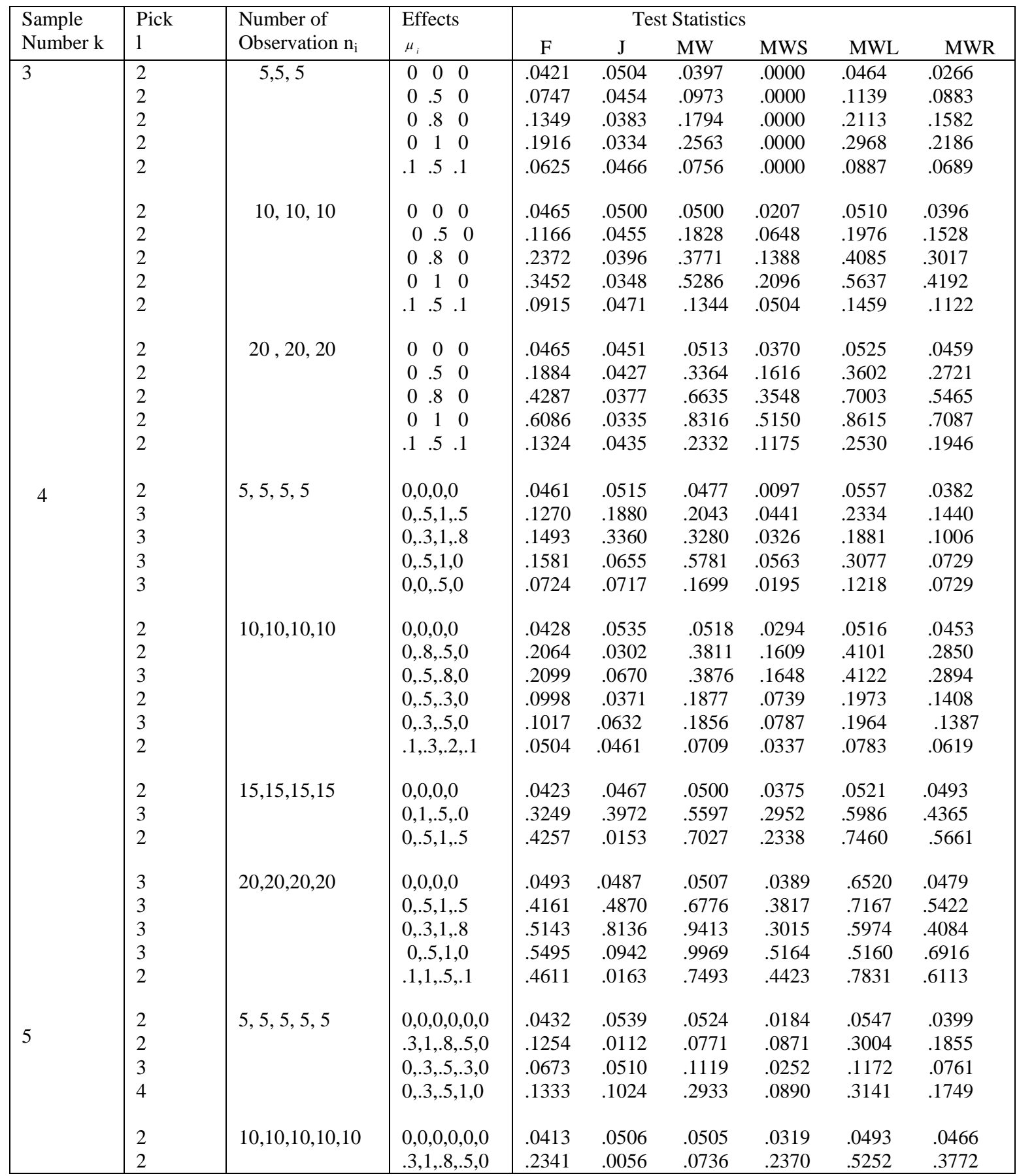


IARJSET

International Advanced Research Journal in Science, Engineering and Technology

Vol. 3, Issue 1, January 2016

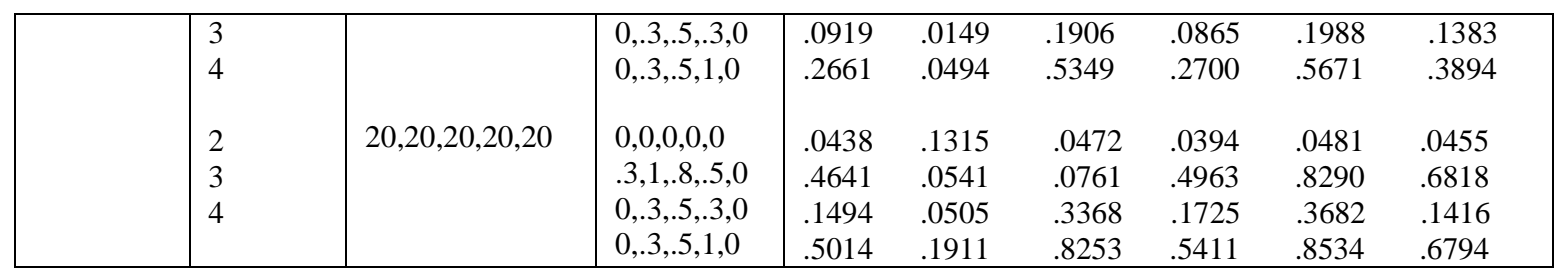

Table 7 Empirical level and power of six tests under Cauchy Distribution

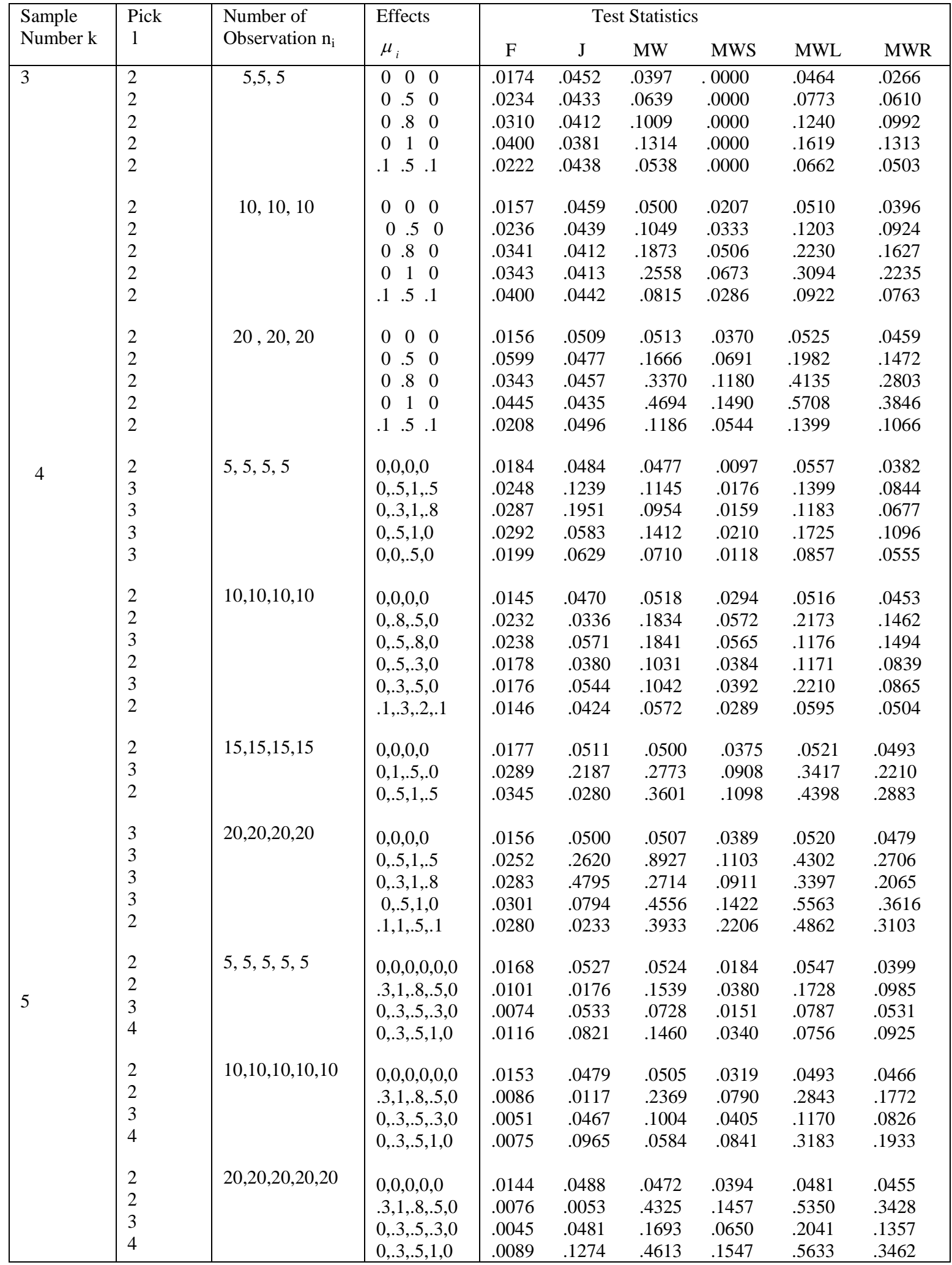




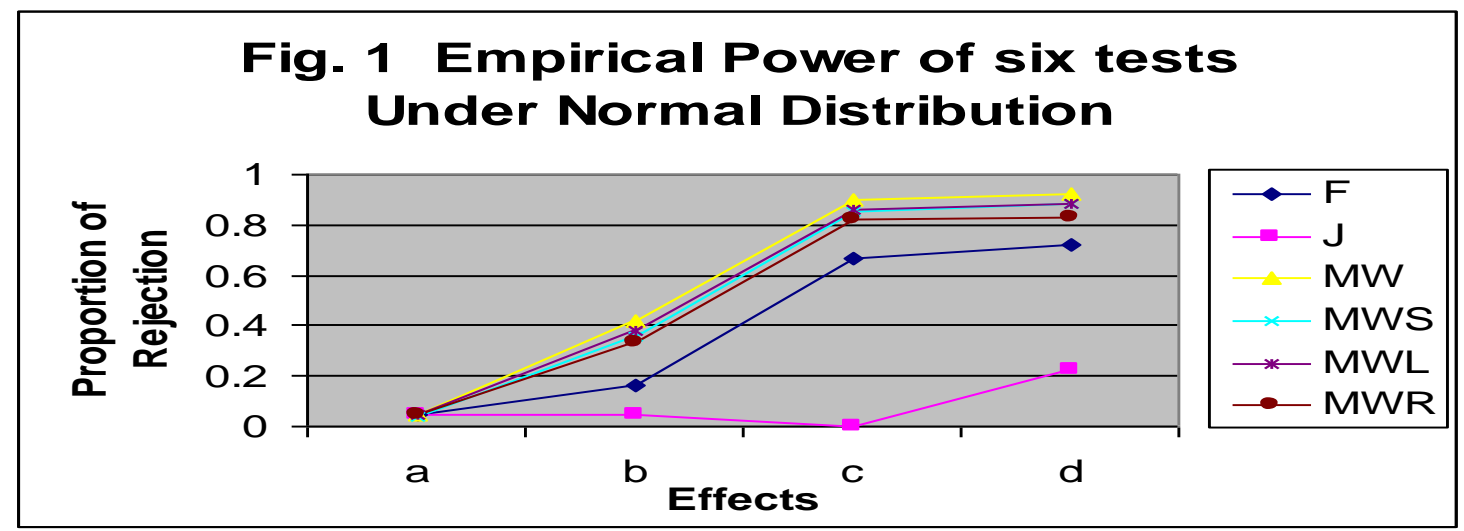

a,b,c,d are level of effects as given in following table

Table 1(i): Level and Power at $\alpha=.05$

\begin{tabular}{|l|l|l|l|l|l|l|l|}
\hline No. of Observation & Effects Type $\mu_{i}$ & F & J & MW & MWS & MWL & MWR \\
\hline a $: 20,20,20,20,20$ & $0,0,0,0,0$ & .0455 & .0484 & .0457 & .0405 & .0470 & .0448 \\
\hline b: $20,20,20,20,20$ & $0, .3, .5, .3,0$ & .1627 & .0446 & .4188 & .3564 & .3774 & .3313 \\
\hline c: $20,20,20,20,20$ & $.3,1, .8, .5,0$ & .6660 & .0010 & .9015 & .8515 & .8578 & .8182 \\
\hline d: $20,20,20,20,20$ & $0, .3, .5,1,0$ & .7197 & .2211 & .9234 & .8824 & .8868 & .8280 \\
\hline
\end{tabular}

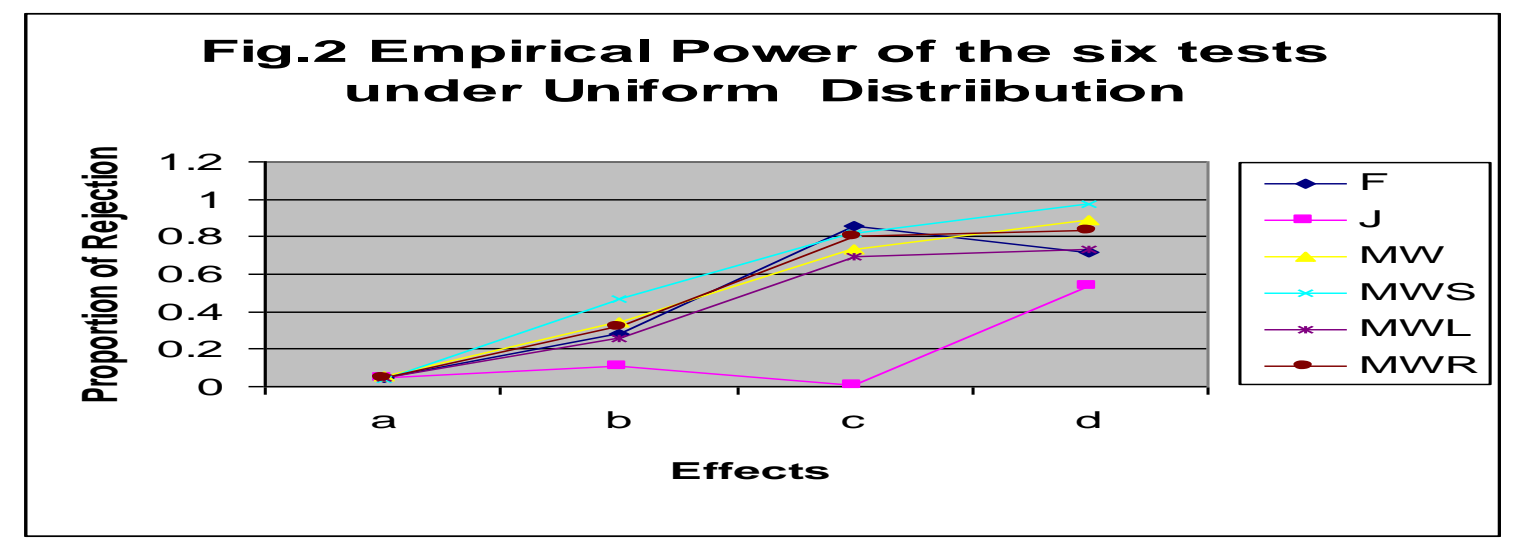

a,b,c,d are level of effects as given in following table

Table 2(i): Level and Power at $\alpha=.05$

\begin{tabular}{|l|l|l|l|l|l|l|l|}
\hline No. of Observation & Effects Type & F & J & MW & MWS & MWL & MWR \\
\hline a: $20,20,20,20,20$ & $0,0,0,0,0$ & .0473 & .0478 & .0518 & .0382 & .0481 & .0455 \\
\hline b:20,20,20,20,20 & $0, .3, .5, .3,0$ & .2818 & .1057 & .3395 & .4664 & .2591 & .3184 \\
\hline c: $20,20,20,20,20$ & $.3,1, .8, .5,0$ & .8605 & .0076 & .7355 & .8214 & .6961 & .7989 \\
\hline d:20,20,20,20,20 & $0, .3, .5,1,0$ & .7152 & .5382 & .8861 & .9721 & .7342 & .8319 \\
\hline
\end{tabular}

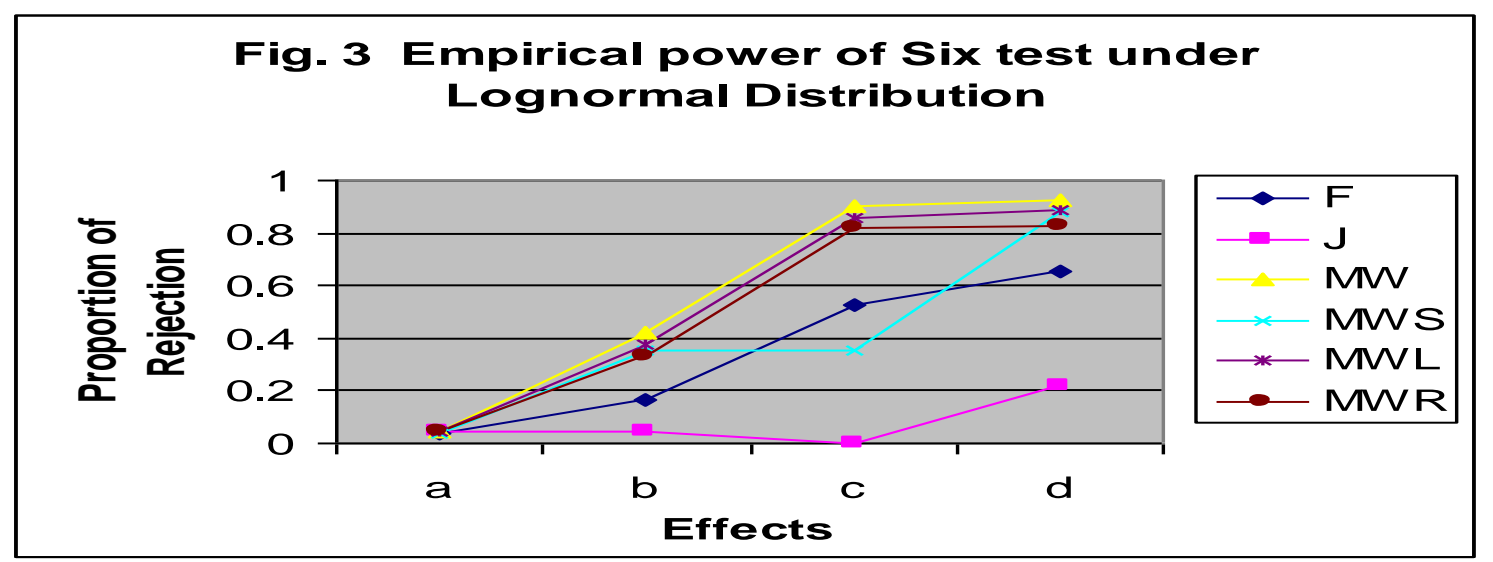

a,b,c,d are level of effects as given in following table 
IARJSET

International Advanced Research Journal in Science, Engineering and Technology

Vol. 3, Issue 1, January 2016

Table 3(i): Level and Power at $\quad \alpha=.05$

\begin{tabular}{|l|l|l|l|l|l|l|l|}
\hline No. of Observation & Effects Type $\mu_{i}$ & F & J & MW & MWS & MWL & MWR \\
\hline a:20,20,20,20,20 & $0,0,0,0,0$ & .0350 & .0484 & .0457 & .0405 & .0470 & .0448 \\
\hline b:20,20,20,20,20 & $0, .3, .5, .3,0$ & .1638 & .0446 & .4188 & .3564 & .3774 & .3313 \\
\hline c: $20,20,20,20,20$ & $.3,1, .8, .5,0$ & .5268 & .0010 & .9015 & .8515 & .8578 & .8182 \\
\hline d: $20,20,20,20,20$ & $0, .3, .5,1,0$ & .6525 & .2211 & .9234 & .8824 & .8868 & .8280 \\
\hline
\end{tabular}

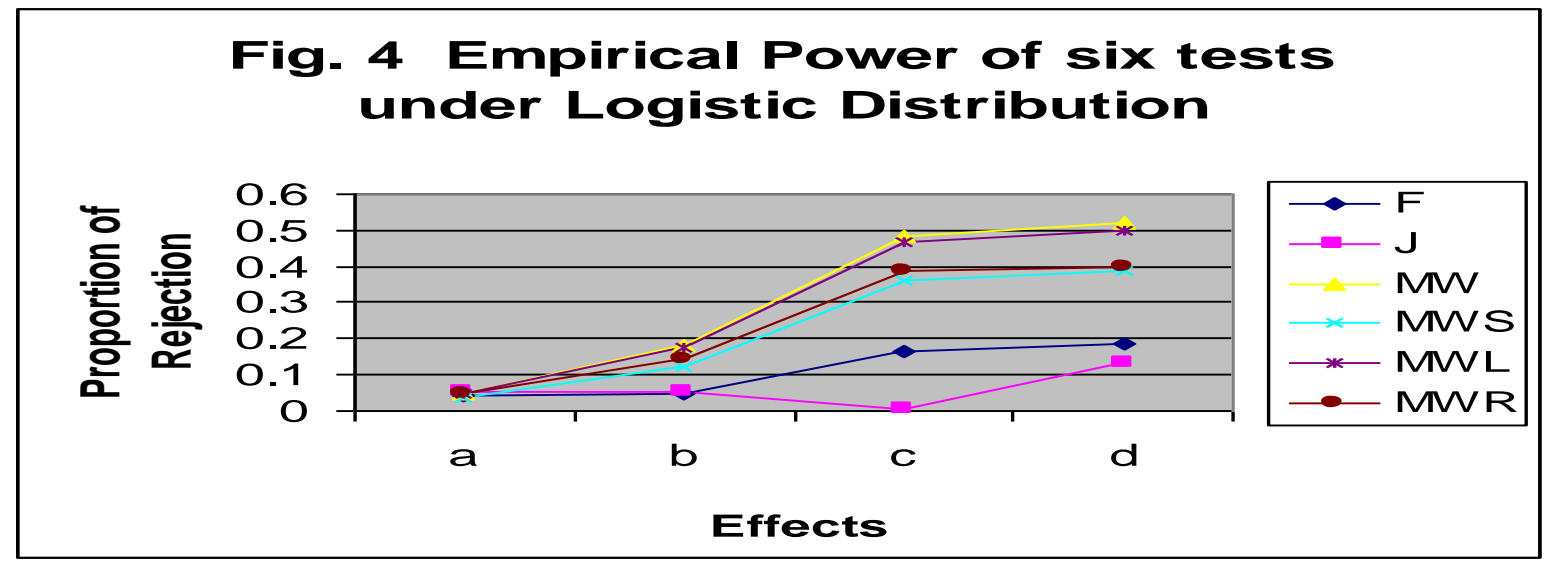

a,b,c,d are level of effects as given in following table

Table 4(i) : Level and Power at $\alpha=.05$

\begin{tabular}{|l|l|l|l|l|l|l|l|}
\hline No. of Observation & Effects Typ $\mu_{i}$ & $\mathrm{~F}$ & $\mathrm{~J}$ & $\mathrm{MW}$ & $\mathrm{MWS}$ & MWL & MWR \\
\hline $\mathrm{a}: 20,20,20,20,20$ & $0,0,0,0,0$ & .0427 & .0541 & .0472 & .0394 & .0481 & .0455 \\
\hline $\mathrm{b}: 20,20,20,20,20$ & $0, .3, .5, .3,0$ & .0482 & .0513 & .1809 & .1242 & .1747 & .1418 \\
\hline $\mathrm{c}: 20,20,20,20,20$ & $.3,1, .8, .5,0$ & .1645 & .0053 & .4856 & .3604 & .4687 & .3882 \\
\hline $\mathrm{d}: 20,20,20,20,20$ & $0, .3, .5,1,0$ & .1879 & .1327 & .5177 & .3851 & .5000 & .3978 \\
\hline
\end{tabular}

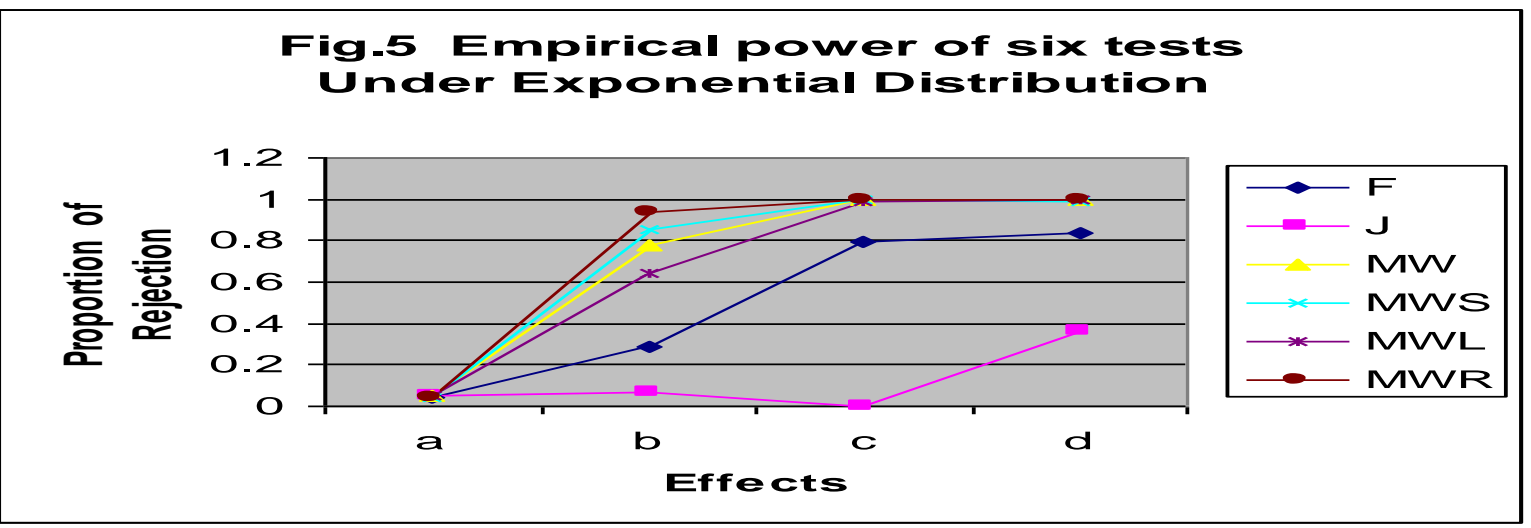

a,b,c,d are level of effects as given in following table

Table 5(i): Level and Power at $\alpha=.05$

\begin{tabular}{|l|l|l|l|l|l|l|l|}
\hline No. of Observation & Effects Type $\mu_{i}$ & F & J & MW & MWS & MWL & MWR \\
\hline a:20,20,20,20,20 & $0,0,0,0,0$ & .0427 & .0541 & .0472 & .0394 & .0481 & .0455 \\
\hline b:20,20,20,20,20 & $0, .3, .5, .3,0$ & .2902 & .0696 & .7772 & .8543 & .6454 & .9399 \\
\hline c:20,20,20,20,20 & $.3,1, .8, .5,0$ & .7925 & .0002 & .9949 & .9941 & .9849 & .9998 \\
\hline d:20,20,20,20,20 & $0, .3, .5,1,0$ & .8385 & .3674 & .9975 & .9919 & .9953 & 1.000 \\
\hline
\end{tabular}




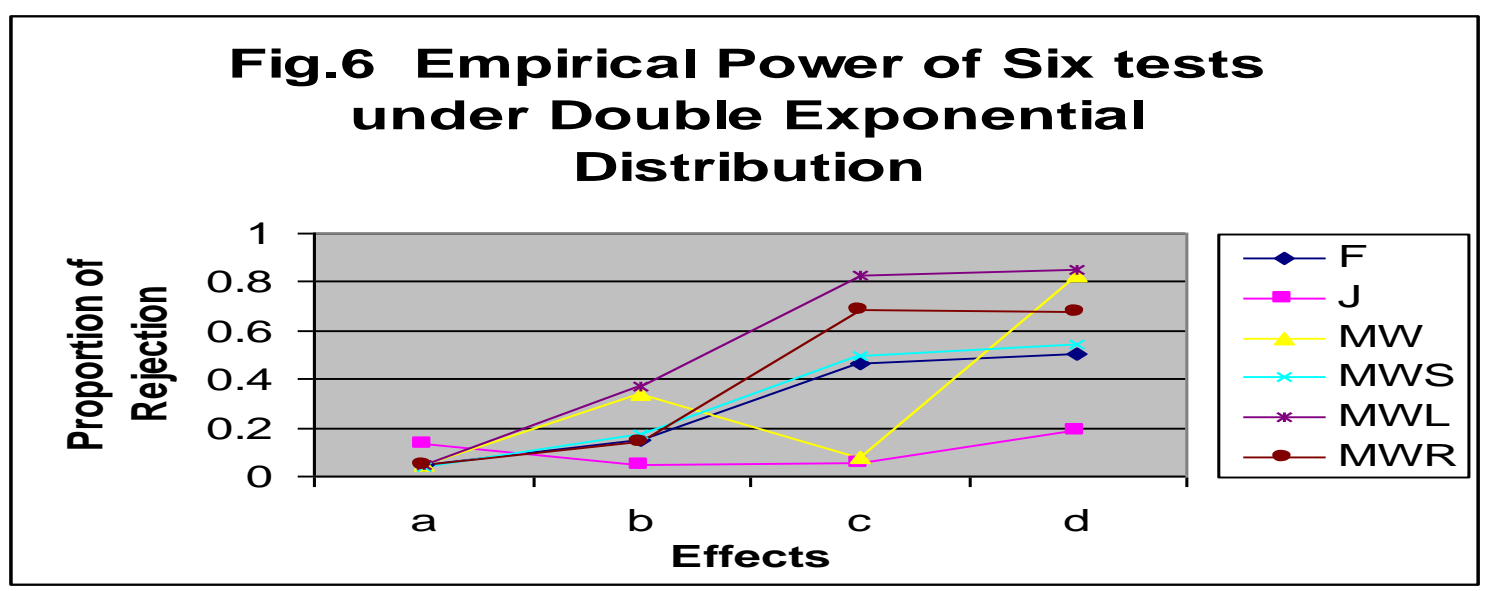

$\mathrm{a}, \mathrm{b}, \mathrm{c}, \mathrm{d}$ are level of effects as given in following table

Table 6(i): Level and Power at $\alpha=.05$

\begin{tabular}{|l|l|l|l|l|l|l|l|}
\hline No. of Observation & Effects Type $\mu_{i}$ & F & J & MW & MWS & MWL & MWR \\
\hline a:20,20,20,20,20 & $0,0,0,0,0$ & .0438 & .1315 & .0472 & .0394 & .0481 & .0455 \\
\hline b:20,20,20,20,20 & $0, .3, .5, .3,0$ & .1494 & .0505 & .3368 & .1725 & .3682 & .1416 \\
\hline $\mathrm{c}: 20,20,20,20,20$ & $.3,1, .8, .5,0$ & .4641 & .0541 & .0761 & .4963 & .8290 & .6818 \\
\hline $\mathrm{d}: 20,20,20,20,20$ & $0, .3, .5,1,0$ & .5014 & .1911 & .8253 & .5411 & .8534 & .6794 \\
\hline
\end{tabular}

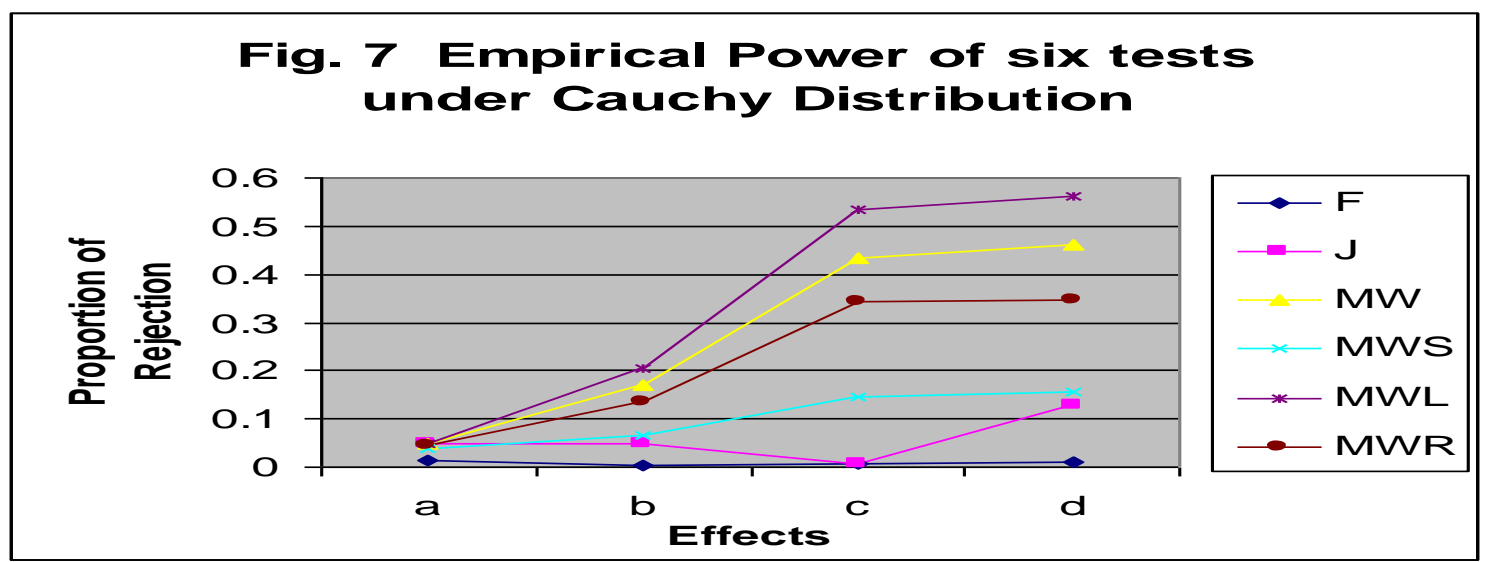

a,b,c,d are level of effects as given in following table

Table 7(i): Level and Power at $\alpha=.05$

\begin{tabular}{|l|l|l|l|l|l|l|l|}
\hline No. of Observation & Effects Type $\mu_{i}$ & F & J & MW & MWS & MWL & MWR \\
\hline a:20,20,20,20,20 & $0,0,0,0,0$ & .0144 & .0488 & .0472 & .0394 & .0481 & .0455 \\
\hline b:20,20,20,20,20 & $0, .3, .5, .3,0$ & .0045 & .0481 & .1693 & .0650 & .2041 & .1357 \\
\hline c:20,20,20,20,20 & $.3,1, .8, .5,0$ & .0076 & .0053 & .4325 & .1457 & .5350 & .3428 \\
\hline d:20,20,20,20,20 & $0, .3, .5,1,0$ & .0089 & .1274 & .4613 & .1547 & .5633 & .3462 \\
\hline
\end{tabular}

\section{DISCUSSION OF THE RESULTS}

We observe from the Table 1 and Fig. 1 that if the peak of levels at various combinations of sample, sample sizes and the umbrella properly chosen, the power of the Mack- pick points. We have found that power of Mack-Wolfe Wolfe test is excellent under the normal distribution. We short tailed test seems to slightly more than Mack Wolfe also observed that power of other Mack-Wolfe type tests test in some situations and far higher than other tests under also quite satisfactory in compared to the F-test and this distribution. Since Uniform distribution is a short Jonchkeere test under this distribution. tailed distribution so it is obvious, because short tailed test Table 2 and Fig.2 give the empirical level and power of based on the scores specially suited for the short tailed the test statistics under uniform distribution. It is observe distribution.

that except the F-test all other test satisfies the nominal

In Table 3 and Fig. 3 we have presented the empirical 
Level and power of the test statistics under lognormal 7 . distribution. We have seen that empirical level of F- test and Mack-Wolfe short tailed test is not satisfactory. Both the tests seems to be conservative in this distribution. Other tests satisfy the levels more or less. While consider the power of the tests, we observed that power of MackWolfe and Mack-Wolfe right skewed test is more than the other test in all the situations considered in this section.

Table 4 and Fig. 4 displayed the empirical level and power of the test statistics under the logistic distribution. We have seen that power of Mack-Wolfe test is the highest of all the test considered for the study. Followed by MackWolfe Long tailed and right skewed test. Power of other test seems to be lower than these three tests.

Level and power of the tests under the exponential distribution are shown in Table 5 and Fig. 5 . It is seen that except the short tailed test all other test satisfies the nominal levels under the null hypothesis. We have noticed that power of right -skewed test is more than other test in this distribution. It is followed by Mack-Wolfe test and Mack-Wolfe long tailed test. Powers of other tests are less than these three tests. Table 6 and Fig. 6 displayed the level and power of the tests under the double exponential distribution. It is seen that power of long tailed test is the highest of the entire test. Next highest power exhibits by Mack-Wolfe test. Then it is followed by Mack-Wolfe right skewed test and short tailed test and F test respectively. Power of other tests is less than the earlier tests.

Finally, we have displayed the empirical level and power of the tests under the Cauchy distribution in Table 7 and Fig.7. Under this distribution F-test and Mack-Wolfe short tailed test not satisfied the nominal levels. While consider the power of the test we have seen than Mack-Wolfe test exhibits the highest power among all the test. Next highest power shown by Mack-Wolfe test followed by MackWolfe right skewed test. Power of other tests is much less than the above tests.

\section{CONCLUSION}

In conclusion we can say that if prior information regarding the peak of the umbrella is known it will help to choose the appropriate test statistic. However, overall performance of Mack-Wolfe (MW) test seems to the best. So, we can recommend this test for all distributions. If prior information regarding the form of the distribution are known, one can choose the specific type of MW-Type of test statistic (discuss above) suitable for that situation

\section{REFERENCES}

1. Archambault,W.A.T.Jr., Mack,G.A. and Wolfe, D.A. (1978) : KSample Rank Tests Using Pair-Specific Scoring Functions, Canadian Jour. Stat., 5, 95-207.

2. Boyd, M.N. and Sen, P.K. (1983): Union-Intersection rank tests for ordered

3. Alternatives in some simple linear models. Comm. Statist. Theor. Meth. 12, 1737-1753.

4. Jonckheere, A.R.(1954): A Distribution-Free k-Sample Tests Against Ordered

5. Alternatives Biometrika, 41, 133-145.

6. Bunning,H. and Kossler,W.(1997): Power of some tests for umbrella alternatives in multi-sample location problem, Biometrical journal, 39, 481-494.
Buning,H. and Kossler, W. (1999): The asymptotic power of Jonckheere-Type Tests for Ordered Alternatives, Austral. And Ne Zealand Statist. 41(1),67-77.

8. Gastwirth,J.L. (1965): Percentile modifications of two-sample rank tests, Jour.Amer.Stat.,Assoc.,1127-1140.

9. Guohua,P.(1996): Distribution-Free tests Umbrella Alternatives, Commun.Statist-

10. Theory Meth.,25(2),3185-3196.

11. Hajek, J. (1968): Asymptotic normality of simple linear rank statistics under

12. alternatives, Ann. Math. Statist. 39,325-345.

13. Hogg, R.V. Fisher, D.M. and Randles, R. H. (1975): A two-sample adaptive

14. distribution- free test, Jour. Amer. Stat. Assoc. 70,656-661.

15. Kruskal, W.H. and Wallis,W.A.(1952): Use of Ranks in OneCriterion Variance

16. Analysis, Jour. Amer. Stat. Assoc. 47,583-621.

17. Mack,G.A. and Wolfe,D.A. (1981): K-Sampe rank tests for umbrella alternatives, Jour.Amer.Stat. Assoc. 76,175-181.

18. Mann, H.B. and Whitney, D.R. (1947): On a test of whether one of two random

19. variables is stochastically larger than the other. Ann. Math Stat. 18,50-60.

20. Puri, M.L. (1965): Some Distribution-free K-sample Rank Tests of Homogeneity Against Ordered Alternatives. Commun. Pure and Applied Math. 18, 51-63.

21. Terpstra,T.J. (1952): The asymptotic normality and consistency of Kendall's test against trend, when ties are present in one ranking. Indag. Math. 14, 327-333.

22. Wechsler, D. (1958): The measurement and Appraisal of Adult Intelligence, The

23. Williams and Wilk Co. Baltimore.

24. Randles,R.H. and Wolfe,D.A.(1979): Introduction to the theory of Nonparametric Statistics, Wiley, New York. 